\title{
Die Verhältnismäßigkeit der Covid-19-Maßnahmen aus strafrechtlicher Sicht
}

\author{
Alois Birklbauer
}

Der vorliegende Beitrag beschäftigt sich mit der Frage, inwieweit die strafrechtlichen Covid-19-Maßnahmen als verhältnismäßig einzustufen sind, insbesondere mit Blick auf den Ultima-Ratio-Grundsatz, der dem Strafrecht die Rolle des letzten Mittels zuschreibt, um die Ordnung in einem Staat aufrechtzuerhalten. Nach einer Einleitung (1.) werden Aspekte des materiellen Strafrechts (2.) sowie ausgewählte Maßnahmen aus dem Strafverfahrensrecht (3.) und Strafvollzugsrecht (4.) kurz dargestellt. In zusammenfassenden Schlussfolgerungen wird mit Blick auf die Möglichkeit der Verhaltenssteuerung durch Strafnormen abschließend die Frage der Verhältnismäßigkeit beurteilt (5.).

\section{Einleitung}

Wie die COVID-19-Krise verdeutlicht hat, verlangen Menschen in unsicheren Zeiten nach Regeln, die ihnen Sicherheit vermitteln. Je größer eine Krise, desto mehr scheint das Bedürfnis zu steigen, durch die Krise geleitet zu werden. Dabei kommt nicht nur Regeln, sondern auch Führungspersonen die Aufgabe zu, Orientierung zu vermitteln. Manche Personen gehen in dieser Aufgabe gleichsam auf und verlangen nach mehr Kompetenzen, um als „starke Männer“ das Volk leiten zu können. Dass dies eine Demokratie, in der das Recht vom Volk ausgeht (vgl. Art. 1 Bundes-Verfassungsgesetz $[\mathrm{B}-\mathrm{VG}]^{1}$ ), an seine Grenzen bringen kann, wenn Gesetzgebungsverfahren verkürzt werden, um die Beteiligung der verschiedenen Parlamentsabgeordneten, die für unterschiedliche Ideen und Werthaltungen einer Gesellschaft stehen, zurückzuschrauben, ist die Kehrseite. Die Verlockung für Regierungen, sich von den Parlamenten Not(verordnungs)rechte geben zu lassen, um Normschaffungsprozesse zu beschleunigen, ist groß. Verschiedene Länder sind unterschiedlich mit solchen Verlockungen um-

1 Bundes-Verfassungsgesetz (B-VG), Bundesgesetzblatt (BGBl.) 1/1930. 
gegangen. Während die einen zur Bedachtsamkeit mahnten (z. B. Deutschland), schalteten andere die Parlamente nahezu völlig aus (z. B. Ungarn). Österreich hat einen Mittelweg gewählt, indem viel darangesetzt wurde, die Krise möglichst dramatisch aussehen zu lassen, damit das Parlament der - zumindest schleichenden - Machtverschiebung an die Regierung möglichst einhellig zustimmt und das Volk dies nicht allzu kritisch hinterfragt.

Das materielle Strafrecht blieb im Rahmen der COVID-19-Krise von Neuerungen verschont. Dies liegt zum einen daran, dass bereits ausreichende Strafnormen vorhanden waren, um die individuelle Gesundheit und die so genannte „Volksgesundheit“ vor Gefährdung zu schützen. Zum andern ist eine rasche Reaktion auf Normverstöße im Bereich des Strafrechts dadurch erschwert, dass es eine gewisse Zeit dauert, bis ein Urteil erfolgen kann und überdies Verurteilungen von einem unabhängigen Gericht erfolgen müssen. Da ist es verlockender, im Rahmen des Verwaltungsstrafrechts Verbote einzuführen, weil hier einerseits rasch über Normverstöße durch (erstinstanzliche) weisungsgebundene Behörden entschieden werden kann und erst im Falle eines Rechtsmittels ein unabhängiges (Verwaltungs-)Gericht sich der Sache annimmt. Andererseits ist die Sanktion im Verwaltungsstrafrecht letztlich fühlbarer als im (niederschwelligen) Kriminalstrafrecht, gibt es doch im Verwaltungsbereich keine bedingten Strafen und sind die Geldstrafen infolge deren Ausgestaltung als Geldsummenstrafe weniger sozial gerecht als die von den Strafgerichten nach dem Tagessatzsystem $^{2}$ zu verhängenden Geldstrafen. Verwaltungsstrafen treffen daher sozial Schwache tendenziell härter als finanziell besser gestellte Personen. Die Reduktion der Verbotsnormen im Rahmen der COVID-19-Krise auf das Verwaltungsstrafrecht war damit unter dem Gesichtspunkt der sozialen Gerechtigkeit nicht unproblematisch. ${ }^{3}$

Der Schutz von Menschen vor Ansteckung mit dem COVID-19-Virus führte zu zahlreichen Änderungen im Strafverfahrensrecht sowie im Bereich des Strafvollzugs, vorwiegend um Kontakte zwischen den Gerichten und den Verfahrensbeteiligten bzw. den Strafgefangenen und dem Justizwachepersonal einzudämmen. „Social Distancing“ war auch hier das Schlagwort

2 Während bei der Geldsummenstrafe Unrecht und Schuld ebenso in der ausgesprochenen Summe der Geldstrafe ihren Ausdruck finden wie die finanzielle Leistungsfähigkeit des Normabweichlers, wird im Tagessatzsystem differenziert: Die Anzahl der verhängten Tagessätze drückt Unrecht und Schuld aus, die konkrete Höhe des Tagessatzes die wirtschaftliche Leistungsfähigkeit des Rechtsbrechers.

3 Die Geldstrafen für Verstöße gegen die Ausgangsbeschränkungen beliefen sich z. B. auf bis zu 3.600 Euro (vgl. \$3 COVID-19-Maßnahmengesetz, BGBl. I 12/2020). 
der Stunde, um mitunter an Grundrechten und Verfahrensprinzipien zu rüttelten.

\section{Ausreichend vorhandene Normen im materiellen Strafrecht}

Die schon vor der COVID-19-Krise vorhandenen Bestimmungen des materiellen Strafrechts schützen einerseits das Individualrechtsgut Leib und Leben ( $\$ \mathbb{S} 75 \mathrm{ff}$ Strafgesetzbuch $\left.[\mathrm{StGB}]^{4}\right)$, andererseits das Universalrechtsgut Leben und Gesundheit der Allgemeinheit („Volksgesundheit") vor Gefährdung von Menschen durch übertragbare Krankheiten $(\mathbb{S} 178 \mathrm{f} \mathrm{StGB).5}$ Kann über das Individualrechtsgut Gesundheit vom jeweiligen Rechtsgutsträger verfügt werden, wodurch der Verursacher einer Gesundheitsschädigung Straffreiheit erlangt, wenn der Geschädigte sich bewusst der Gesundheitsgefährdung ausgesetzt hat, ist das Universalrechtsgut "Volksgesundheit“ einer strafbefreienden Disposition seitens des Geschädigten von vornherein entzogen.

\subsection{Körperverletzungsdelikte}

Die Körperverletzungsdelikte ( $\mathbb{S} 83 \mathrm{ff}$ StGB) erfassen neben der Beeinträchtigung eines konkreten Individuums in seiner körperlichen Integrität (Unversehrtheit) gleichermaßen die Gesundheitsschädigung als Beeinträchtigung des physischen und/oder psychischen Wohlbefindens, welche Krankheitswert im medizinischen Sinn besitzt. ${ }^{6}$ Dabei wird die Erheblichkeitsschwelle niedrig angesetzt, reicht doch z.B. für eine Körperverletzung ( $\$ 83$ Abs. $1 \mathrm{StGB}$ ) bereits eine nicht allzu große Hautrötung aus, um den Tatbestand zu begründen. ${ }^{7}$ Eine COVID-19-Infektion überschreitet somit, unabhängig von ihrem konkreten Verlauf, die Tatbestandsschwelle für eine Gesundheitsschädigung. Dauert diese mehr als 24 Tage, ist sie als „schwere Körperverletzung“( $\$ 84$ Abs. 1 StGB) einzustufen.

4 Bundesgesetz vom 23. Jänner 1974 über die mit gerichtlicher Strafe bedrohten Handlungen (Strafgesetzbuch - StGB), BGBl. 60/1974.

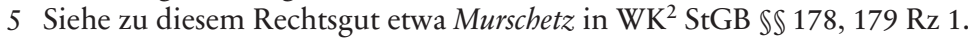

6 Siehe dazu etwa Burgstaller/Fabrizy in WK ${ }^{2}$ StGB $\$ 83 \mathrm{Rz} 9 \mathrm{ff}$.

7 Vgl. OGH 11 Os 180/76 = SSt 48/20: linsengroßer Hautdefekt am Grundgelenk des 5. Fingers links und oberflächliche Hautabschürfungen an der linken Augenbraue. 


\subsection{Gefährdung der „Volksgesundheit“"}

Die Gefährdung von Menschen durch übertragbare Krankheiten $(\mathbb{S} \$ 178 \mathrm{f}$ StGB) setzt zunächst eine geeignete Handlung voraus, um die Gefahr der Verbreitung einer übertragbaren Krankheit unter Menschen herbeizuführen. Dabei gelten als übertragbare Krankheiten jene, bei denen ein Krankheitserreger unmittelbar oder mittelbar von einem Individuum auf ein anderes übergehen kann. ${ }^{8}$ Im Unterschied zu den Körperverletzungsdelikten kommt es hier nicht darauf an, dass tatsächlich jemand durch das Verhalten eines anderen infiziert wird, sondern es reicht aus, dass die Gefahr einer Verbreitung, also eine Zunahme des Ausdehnungsbereichs der Krankheit herbeigeführt wird, ohne dass es epidemischer Ausmaße bedürfe. ${ }^{9}$

Damit die Strafbarkeit nicht zu sehr ausufert, muss die Krankheit ihrer Art nach zu den zumindest beschränkt anzeige- oder meldepflichtigen Krankbeiten gehören, um nicht auch relativ ungefährliche Krankheiten wie Schnupfen oder Grippe unter das Delikt zu subsumieren. ${ }^{10}$ Diese Anzeigebzw. Meldepflicht ist eine objektive Bedingung der Strafbarkeit, ${ }^{11}$ die dem Täter weder bewusst noch für ihn erkennbar gewesen sein muss.

COVID-19 ist eine übertragbare Krankheit, deren Erreger unmittelbar oder mittelbar von einem Individuum auf ein anderes übergehen kann. Die Krankheit ist meldepflichtig nach $\mathbb{1} 1$ Abs. 1 Z. 1 Epidemiegesetz, ${ }^{12}$ wodurch auch die genannte objektive Bedingung der Strafbarkeit als Voraussetzung für eine Subsumtion unter $\mathbb{S} 178 \mathrm{f}$ StGB erfüllt ist.

\subsection{Vorsätzliche und fahrlässige Begehungsweise}

Das Strafrecht erfasst sowohl bei der Körperverletzung als auch der Gefährdung von Menschen durch übertragbare Krankheiten vorsätzliche und fahrlässige Begehungsweisen. Fabrlässigkeit im Sinne von $\$ 6$ StGB setzt die objektive Sorgfaltswidrigkeit eines Verhaltens voraus. Eine solche liegt vor, wenn entweder gegen eine positivierte Rechtsvorschrift (Gesetz oder Ver-

$8 \mathrm{Vgl}$. Leukauf/Steininger/Tipold, StGB 4 \$ $178 \mathrm{Rz} 2$.

9 Vgl. Leukauf/Steininger/Tipold, StGB $\$ 178 \mathrm{Rz} 8 ;$ Murschetz in $\mathrm{WK}^{2}$ StGB $\mathbb{S}$ 178, $179 \mathrm{Rz} 2$.

$10 \mathrm{Vgl}$. Leukauf/Steininger/Tipold, $\mathrm{StGB}^{4} \$ 178 \mathrm{Rz} 5$.

11 Siehe etwa Leukauf/Steininger/Tipold, StGB $\$ 178 \mathrm{Rz} 4 ;$ Murschetz in WK ${ }^{2}$ StGB \\178, $179 \mathrm{Rz} 5$.

12 Epidemiegesetz 1950, BGB1. 186/1950. Die Bestimmung nennt MERS-CoV (Middle East Respiratory Syndrome Coronavirus/„neues Corona-Virus“). 
ordnung) oder eine berufsspezifische Sorgfaltsnorm (leges artis) verstoßen wird. Fehlt es an solchen generellen Verhaltensanordnungen, wird subsidiär auf den einsichtigen und besonnenen Menschen aus dem Verkehrskreis des Täters abgestellt (objektiver Dritter), der sich in der konkreten Situation anders, nämlich weniger riskant verhalten hätte. ${ }^{13} \mathrm{Im}$ Unterschied dazu bedeutet Vorsatz nach der Legaldefinition des $\$ 5$ Abs. 1 StGB die Erfüllung eines strafrechtlichen Tatbestands ernstlich für möglich halten (Wissenskomponente) und sich mit dieser Erfüllung abfinden (Wollenskomponente). Ein Täter, der das mit seinem Verhalten einhergehende objektive Risiko erkennt und dessen Verwirklichung in Kauf nimmt, indem er sein Verhalten in Kenntnis dieses konkreten Risikos setzt, handelt vorsätzlich. ${ }^{14}$

Da die gesundheitlichen Risiken einer COVID-19-Infektion spätestens seit dem Shut Down Mitte März 2020 allgemein bekannt sind, besteht für Personen, die selbst infiziert sind, in den meisten Fällen eine Strafbarkeit wegen vorsätzlicher Körperverletzung ( $\$ \$ \$ 83 \mathrm{ff} S t G B$ ), wenn sie die Kontakte mit anderen Menschen nicht begrenzen und gegen Quarantäneauflagen verstoßen, sofern sich die Kausalität des gesetzten Verhaltens für die Ansteckung eines anderen nachweisen lässt. Bei fehlendem Vorsatz wird zumindest eine fahrlässige Körperverletzung ( $\$ 88$ St GB) naheliegen, denn wer infiziert ist oder infiziert sein könnte und sich nicht an Quarantäneregeln hält, handelt objektiv sorgfaltswidrig, weil er gegen positivierte Normen verstößt. Eine eingetretene Gesundheitsschädigung liegt im Schutzbereich der verletzten Norm.

Schwieriger ist die Strafbarkeit wegen Körperverletzung für jene zu begründen, die sich nicht an die allgemein verordneten Ausgehbeschränkungen halten, die unabhängig von einer eigenen Erkrankung gelten. Hier wird ebenfalls gegen positiviertes Recht verstoßen, dessen Schutzzweck in der Eindämmung des Ansteckungsrisikos mit COVID-19 besteht. Wenn es also gelingt, einen Kausalzusammenhang zwischen einer Infektion und einem konkreten Verursacher, der die Ausgangsbeschränkungen verletzt hat, herzustellen, was mitunter schwierig ist, liegt es nahe, das Verhalten unter den Tatbestand der Körperverletzungsdelikte zu subsumieren.

Für die vorsätzliche Gefährdung mit übertragbaren Krankheiten ( 178 $S t G B)$ muss sich der Vorsatz lediglich auf die Eignung des Verhaltens beziehen, die Gefahr der Verbreitung einer übertragbaren Krankheit unter Men-

13 Siehe zu den Grundzügen der Fahrlässigkeit mit Nachweisen zum Meinungsstand etwa Burgstaller/Schütz in WK ${ }^{2}$ StGB $\$ 6 \mathrm{Rz} 33 \mathrm{ff}$ und $42 \mathrm{ff}$.

$14 \mathrm{Zu}$ dieser Untergrenze des Vorsatzes siehe mit Nachweisen zum Meinungsstand etwa Reindl-Krauskopf in WK $\mathrm{W}^{2} \mathrm{StGB} \$ 5 \mathrm{Rz} 34 \mathrm{ff}$. 
schen herbeizuführen, ${ }^{15}$ was bei den allgemein zugänglichen Informationen über COVID-19 seit März 2020 gegeben ist, wenn es jemand ernstlich für möglich hält und sich damit abfindet, selbst infiziert zu sein. Die subjektive Einschätzung der genannten Infektion als minder gefährlich spielt hier keine Rolle, weil es nur auf die objektive Anzeige- bzw. Meldepflicht ankommt. Nur wenn jemand die eigene Infektion nicht erkennt, wird der für $₫ 178$ StGB erforderliche Vorsatz fehlen.

Eine fahrlässige Gefäbrdung mit übertragbaren Krankheiten (\$ 179 StGB) lässt sich wohl nicht in Fällen begründen, in denen jemand davon ausgeht, selbst infektionsfrei zu sein. Hier kann die bloße Verletzung von Ausgangsbeschränkungen mangels „typischer Gefährdungseignung“ des gesetzten Verhaltens nicht als ausreichend für die Tatbestandsverwirklichung angesehen werden. Dies ergibt sich aus der Stoßrichtung des genannten Delikts, die Gefährdung von Menschen durch ansteckende Krankheiten zu verhindern. Für Verstöße gegen Ausgangsbeschränkungen ist die Sanktionierung nach den verwaltungsstrafrechtlichen Bestimmungen ausreichend. ${ }^{16}$

\section{Maßnahmen im Bereich des Strafprozessrechts}

Das Ziel der COVID-19-Krise, Menschen in ihrem täglichen Lebensbereich vor Ansteckung zu schützen, hat zu wesentlichen Veränderungen im Strafverfahrensrecht geführt, da Verfahren von persönlichen Interaktionen leben. Im Folgenden wird auf die im Rahmen von COVID-19-Maßnahmen erleichterte Möglichkeit von Videovernehmungen sowie die faktische Beschränkung der Öffentlichkeit in der Hauptverhandlung eingegangen, mit Blick auf die Beschränkung von Grundrechten. Besuchsbeschränkungen bei der Untersuchungshaft (U-Haft) werden beim Strafvollzug mitbehandelt. Es soll an dieser Stelle nicht verschwiegen werden, dass es auch Änderungen gab, die Rechtsbrecher begünstigten, wie z.B. die Verlängerung der Fristen zur Erbringung von sanktionsersetzenden Leistungen im $\mathrm{Zu}$ sammenhang mit einer Verfahrenseinstellung (Diversion). Diese werden im vorliegenden Beitrag aus Platzgründen jedoch nicht weiter dargestellt. ${ }^{17}$

15 Vgl. Leukauf/Steininger/Tipold, $\mathrm{StGB}^{4} \mathbb{} 178 \mathrm{Rz} 9$.

16 Siehe dazu Cohen, JSt 2020, S. $204 \mathrm{ff}$.

17 Eingehend zu den weiteren Änderungen in der StPO Birklbauer in Resch, CoronaHB Kap 16 Rz 25 ff. 
Die meisten Änderungen im Bereich des Strafprozessrechts sind durch $\$ 9$ 1. COVID-19-JuBG ${ }^{18}$ ergangen. Dabei erfolgten manche unmittelbar in der StPO und ohne Befristung. Diese können in der Zukunft bei vergleichbar gehäuften Erkrankungen leichter nutzbar gemacht werden, indem nicht mehr auf entsprechende Sondergesetze gewartet werden muss. $\$ 9$ 1. COVID-19-JuBG räumt der Bundesministerin für Justiz (BMJ) weitgehende Verordnungsermächtigungen zur Konkretisierung von bestimmten Maßnahmen ein.

\subsection{Erleichterte Ermöglichung von Videovernehmungen}

Eine zentrale Änderung betrifft die (erleichterte) Vernehmung von Beschuldigten und Zeugen per Video. Diese Möglichkeit wurde als gleichsame Alternative der "innerstaatlichen Rechtshilfe“ bereits im Jahre 2010 in die Strafprozessordnung (StPO) ${ }^{19}$ eingefügt, ${ }^{20}$ allerdings nur für die U-Haft und begrenzt auf den Fall, dass der Aufenthaltsort eines Zeugen oder Beschuldigten außerhalb des Sprengels der zuständigen Staatsanwaltschaft (StA) oder des zuständigen Gerichts liegt und eine unmittelbare Vernehmung verfahrensökonomisch nicht zweckmäßiger erscheint. Im Rahmen der COVID-19-Krise wurde diese Möglichkeit nun auf Fälle einer Pandemie ausgedehnt ( $\left.\mathbb{1 7 4} \mathrm{Abs} .1 \mathrm{Satz} 2 \mathrm{StPO}{ }^{21}\right)$. Videovernehmungen beim Pflichtverhör zur U-Haft-Verbängung wurden damit auch dann zulässig, wenn der Beschuldigte direkt in der Justizanstalt des zuständigen Gerichts angehalten wird. Damit sollen COVID-19-Erkrankungen durch direkten persönlichen Kontakt zwischen Häftlingen, Bediensteten der Justizanstalt und Angehörigen von Gericht und StA möglichst verhindert werden. Mit demselben Ziel wurden Videovernehmungen bei Verhandlungen zur U-HaftVerlängerung ermöglicht ( $\$ 176$ Abs. 3 StPO).

Da die Möglichkeit für derartige Videovernehmungen nach dem Gesetzeswortlaut auf das Vorliegen einer Pandemie oder eine Verordnung der BMJ zur Verhütung und Bekämpfung anzeigepflichtiger Krankheiten beschränkt ist, sollte nach den Vorstellungen des Gesetzgebers die Verhält-

18 Bundesgesetz betreffend Begleitmaßnahmen zu COVID-19 in der Justiz (1. COVID-19-Justiz-Begleitgesetz - 1. COVID-19-JuBG); BGBl. I 16/2020. Dieses Gesetz tritt gemäß seinem $\ 12$ mit 31.12.2020 außer Kraft.

19 Strafprozeßordnung 1975 (StPO); BGBl. 1975/631.

20 BGBl. I 111/2010.

21 BGBl. I 14/2020. 
nismäßigkeit gewahrt werden, ${ }^{22}$ zumal eine derartige Verordnung meist nur zeitlich befristet gelten wird und damit letztlich keine von Krisenfällen unabhängige Erweiterung der StPO erfolgt. Eine solche Verordnung ist mittlerweile ergangen, ${ }^{23}$ wobei die darin ursprünglich vorgesehene Verpflichtung zur Videovernehmung bereits tags darauf in eine schlichte Möglichkeit umgewandelt wurde. ${ }^{24}$ Damit lag die Entscheidung gegen eine Videovernehmung letztlich im Ermessen des Gerichts, ohne dass jedoch die Verordnung dafür Kriterien normierte.

Besteht grundsätzliches Verständnis für die Erweiterung der Möglichkeiten einer Videovernehmung im Zusammenhang mit der COVID-19Krise, gilt dies weniger für die ebenfalls getroffene Veränderung, Entscheidungen über die Fortdauer der U-Haft obne Haftverhandlung und damit ohne Vernehmung des Gefangenen (zumindest per Video) zu ermöglichen. ${ }^{25}$ Hier scheint der Bequemlichkeitsgedanke eine Rolle gespielt zu haben, denn eine Ansteckung mit COVID-19 kann auch durch eine Videovernehmung verhindert werden und braucht keinen Verhandlungsverzicht. Die Bedenken gegen diese Erweiterung werden noch dadurch gestützt, dass nähere Kriterien für einen Verhandlungsverzicht fehlen. Dem wurde zwar in einer Novellierung von $\$ 9 Z 4$ 1. COVID-19-JuBG durch Ergänzung um den Satz: „soweit im Einzelfall eine Durchführung der Haftverhandlung unter Verwendung technischer Einrichtungen zur Wort- und Bildübertragung nicht möglich ist" ${ }^{26}$ versucht, abzuhelfen, eine ausreichende $\mathrm{Be}$ stimmtheit wohnt aber auch dieser Neufassung nicht inne. Damit könnte es für einen Verhandlungsverzicht sowohl ausreichend sein, dass der zuständige Richter im Home-Office arbeitet, in freiwilliger Quarantäne ist oder über kein leistungsfähiges Internet verfügt, mit dem er eine Videovernehmung durchführen könnte, als auch, dass sich der zuständige Richter mit der entsprechenden Technik nicht auseinandersetzen will und deshalb auf eine Videovernehmung verzichtet. Sachgerecht erscheint diese Unbe-

22 Vgl. Bericht des Budgetausschusses des Nationalrates, Nr. 104 der Beilagen zu den Stenographischen Protollen des Nationalrats (BlgNR) 27. Gesetzgebungsperiode (GP) S. 1.

23 Die ursprüngliche Verordnung der BMJ vom 16.3.2020 (BGBl. II 99/2020) wurde von der Verordnung BGBl. II 113/2020 abgelöst. Sie tritt entsprechend ihrem $₫ 8$ mit 30.9.2020 außer Kraft (BGBl. II 243/2020).

24 Vgl. BGBl. II 114/2020.

25 Hierzu räumte $\$ 9 \mathrm{Z} 4$ 1. COVID-19-JuBG der BMJ eine Verordnungsermächtigung ein, von der sie durch BGBl. II 113/2020 Gebrauch gemacht hat. Die entsprechende Bestimmung der Verordnung tritt nach ihrem $\$ 8$ mit 30.9.2020 außer Kraft (BGBl. II 243/2020).

26 BGBl. I. 24/2020 Art 32 Z 7. 
stimmtheit mit Blick auf den Verlust an rechtsstaatlichen Garantien, die eine Vernehmung beinhaltet, nicht.

Videovernehmungen wurden zur Verhinderung von COVID-19Infektion auch für die Hauptverhandlung ermöglicht. ${ }^{27}$ Nach $\$ 239$ Satz 2 StPO ${ }^{28}$ braucht ein verhafteter Angeklagter nicht mehr zur Hauptverhandlung vorgeführt werden, sondern er kann dieser per Videoschaltung folgen und sich auch per Video einbringen, wodurch seine Beschuldigtenrechte gewahrt bleiben (sollten). Eine Einschränkung wurde mit Wirkung 1. Juni 2020 dahingehend getroffen, dass im Geschworenenverfahren ein solches Vorgehen nur dann zulässig ist, wenn „es im Einzelfall besonders gewichtige Gründe für unabdingbar erscheinen lassen“29.

Bei allem Verständnis für die Nutzung neuer technischer Möglichkeiten darf bei näherer Betrachtung nicht übersehen werden, dass damit ein beträchtlicher Verlust an Verteidigungsmöglichkeiten einher gehen kann. Dies erscheint insofern wenig bedacht worden zu sein, als sich nirgends eine Regelung findet, wie etwa in Fällen einer Videovernehmung mit der Beiziehung eines Verteidigers umzugehen ist und wo er sich bei der Vernehmung befinden soll. Naheliegend wäre es, dass der Verteidiger beim Beschuldigten in der Haftanstalt sitzt (in entsprechendem Abstand und unter Einhaltung der erforderlichen Sicherheitsbestimmungen), denn eine bloße Zuschaltung des Verteidigers per Video wird vielfach nicht ausreichen, weil auf diese Weise eine unkontrollierte Besprechung zwischen Verteidiger und Mandant nicht gewährleistet ist. ${ }^{30}$ Für die Hauptverhandlung bedeutet dies freilich, dass die unmittelbare Möglichkeit, sich in die Verhandlung einzubringen, verloren geht, wenn der Verteidiger nicht unmittelbar vor Ort ist. Dies könnte die Qualität der Verteidigung beeinträchtigen. Insofern wäre es besser, der Verteidiger würde im Verhandlungssaal sitzen, wodurch aber wiederum eine unumschränkte Kommunikation mit seinem Mandanten nur bei einer Unterbrechung der Hauptverhandlung möglich wäre, im Zuge derer der Verteidiger seinen Mandanten in der Jus-

$27 \mathrm{Zu}$ den ebenfalls geschaffenen Möglichkeiten von Videovernehmungen im Rechtsmittelverfahren siehe Birklbauer in Resch, Corona-HB Kap 16 Rz 51 ff.

28 BGBl. I 14/2020.

$29 \$ 4$ der Verordnung der BMJ zur Verhinderung der Verbreitung von COVID-19; BGBl. II 113/2020 in der Fassung 243/2020.

30 Laut Mitteilung von BMJ Zadić gegenüber dem Parlament wurde auf die Kritik von Rechtsanwälten, dass Angeklagte bei Videoverhandlungen nicht immer die Möglichkeit hätten, sich ungestört mit ihrem Verteidiger zu unterhalten, insofern reagiert, als U-Häftlingen ein Handy zur Verfügung zu stellen ist, damit sie sich ungestört - mit ihren Verteidigern unterhalten können (Parlamentskorrespondenz Nr. 372 vom 24.04.2020). 
tizanstalt aufsucht. Diese Überlegungen zeigen mit Blick auf den Verhältnismäßigkeitsgrundsatz und Grundrechtsüberlegungen, dass Hauptverhandlungen per Videokonferenz die absolute Ausnahme bleiben müssen und einer Vertagung von Verhandlungen auf Zeiten nach den COVID-19Beschränkungen der Vorzug zu geben ist, was jedoch mit Blick auf das Beschleunigungsgebot, das für Haftsachen gilt, problematisch sein kann.

\subsection{Faktische Beschränkung öffentlicher Hauptverhandlungen}

Art 90 Abs. 1 B-VG normiert die Verpflichtung zur Öffentlichkeit von Verhandlungen in Strafsachen mit dem Vorbehalt, dass Ausnahmen das Gesetz bestimmt. In ähnlicher Weise kennt Art. 6 Abs. 1 der Europäischen Menschenrechtskonvention (EMRK) ${ }^{31}$ den Öffentlichkeitsgrundsatz. Sinn dessen ist es, sowohl eine (demokratische) Kontrolle der Justiz zu ermöglichen als auch den Angeklagten vor einer überbordenden Gerichtsbarkeit zu schützen. ${ }^{32}$

In Umsetzung dieser Verfassungsnormen lässt die StPO Einschränkungen der Öffentlichkeit zu, wobei jedoch die Verkündung des Urteils immer öffentlich erfolgen muss ( $\$ 229$ Abs. 4 StPO). Für die Verhandlung kann die Öffentlichkeit unter anderem wegen Gefährdung der öffentlichen Ordnung oder der nationalen Sicherheit ausgeschlossen werden $(\mathbb{} 229$ Abs. 1 Z. 1 StPO). Ob das Ansteckungsrisiko bei einer Pandemie darunter zu subsumieren ist, bleibt fraglich. ${ }^{33}$ Und selbst bei einem Ausschluss der Öffentlichkeit dürfen Richter, StA und Strafverteidiger, auch wenn sie mit dem konkreten Verfahren nichts zu tun haben, niemals ausgeschlossen werden. Zudem dürfen Angeklagte, Opfer und Privatbeteiligte verlangen, dass drei Personen ihres Vertrauens der Zutritt gestattet werde (vgl. $\mathbb{} 230$ Abs. 2 StPO). Dadurch soll stets ein Mindestmaß an Kontrolle der Justiz gewährleistet bleiben.

Nun hat es der Gesetzgeber im Zuge der COVID-19-Gesetze nicht gewagt, in den verfassungsrechtlich gewährleisteten Öffentlichkeitsgrundsatz einzugreifen. Selbst wenn verhaftete Angeklagte per Video vernommen werden (vgl. $\$ 239$ Satz 2 StPO), durften Zuhörer der Hauptverhandlung

31 Konvention zum Schutze der Menschenrechte und Grundfreiheiten; BGBl. 210/1958.

32 Siehe dazu mit Nachweisen etwa Birklbauer, JSt 2009, S. 109; weiters Danek/Mann in WK-StPO $\$ 228 \mathrm{Rz} 4$.

33 Siehe dazu die Beispiele bei Danek/Mann in WK-StPO $\$ 229 \mathrm{Rz} 2 \mathrm{f}$, welche allgemeine Gesundheitserwägungen nicht zu tragen vermögen. 
beiwohnen. Allerdings hat dies die entsprechende Verordnung des Gesundheitsministers, welche infolge des $\$ 2$ COVID-19-Maßnahmengesetzes $^{34}$ ergangen ist ${ }^{35}$, zumindest entsprechend der Darstellung seitens der Bundesregierung nicht zugelassen. Bei den in $\$ 2$ normierten Ausnahmen vom Verbot des Betretens öffentlicher Orte war die Wahrung von Grundrechten oder die Inanspruchnahme von Grundsätzen der Demokratie, wie sie z. B. hinter dem Öffentlichkeitsgrundsatz stehen, nicht angeführt. Insofern wäre es nicht erlaubt gewesen, das Haus zu verlassen, um einer öffentlichen Gerichtsverhandlung als Zuhörer beizuwohnen. Überspitzt formuliert war eine Verhandlung zwar de jure weiterhin öffentlich, de facto aber infolge der genannten Verordnung nicht, weil der Weg zum Gericht keine Ausnahme vom Verbot, öffentliche Orte zu betreten, bildete. Der Gesundheitsminister hatte somit gleichsam per Verordnung den verfassungsrechtlichen Öffentlichkeitsgrundsatz untergraben. Unabhängig von der Beurteilung, ob eine derartige Verordnung überhaupt verfassungsrechtlich gedeckt ist, sollte die Möglichkeit, Verfassungsgrundsätze derart leicht aus den Angeln zu heben, unter Verhältnismäßigkeitsaspekten nachdenklich stimmen. ${ }^{36}$

Freilich war auch eine andere Lesart der Verordnung möglich, welche die Regierungsspitze rasch als „Interpretation spitzfindiger Juristen“ bezeichnet hat, obwohl sie sich nur am Wortlaut der Norm orientierte, wie es Juristen am Beginn ihres Studiums lernen. $\$ 2$ Z. 5 der genannten Verordnung nannte als Ausnahme vom Verbot des Betretens öffentlicher Orte im Freien, wenn diese „alleine, mit Personen, die im gemeinsamen Haushalt leben, oder mit Haustieren betreten werden sollen, gegenüber anderen Personen ist dabei ein Abstand von mindestens einem Meter einzuhalten“. Ein bestimmter Zweck des Betretens war darin nicht genannt und damit nicht erforderlich. Sportliche Betätigung, Spazierengehen oder schlichter Aufenthalt im Freien zum Sonnenbaden waren damit ebenso zulässig wie der Weg zu Freunden oder auch zum Gericht, um einer Ladung Folge zu leisten oder einer Verhandlung beizuwohnen. Das immer wieder seitens der Regierung ins Spiel gebrachte Verbot, andere Menschen zu besuchen, mag zwar zur Eindämmung der Pandemie vernünftig gewesen sein, es fand aber weder in den entsprechenden Gesetzen noch in den dazu ergangenen Verordnungen seine Deckung.

34 BGBl. I 12/2020.

35 BGBl. II 98/2020 in der Fassung 162/2020. Diese Verordnung ist entsprechend ihrem $\$ 7$ mit 30.4.2020 außer Kraft getreten.

36 Kritisch zu dieser Maßnahme Birklbauer, JSt 2020, S. 197. 
Für die Öffentlichkeit der Hauptverhandlung war aus der interpretierbaren Zulässigkeit, zum Gericht zu gelangen, jedoch mitunter wenig gewonnen, weil potentielle Zuhörer es zwar bis zum Gericht schafften, aber nicht in das (verschlossene) Gebäude gelangten bzw. die Sicherheitskontrolle eine für sie unüberwindliche Schranke bildete. Damit kam es faktisch infolge der COVID-19-Maßnahmen zumindest in den ersten Wochen nach dem Shut Down zu keinen öffentlichen Hauptverhandlungen, obwohl Hauptverhandlungen durchgeführt wurden. Dies ist unter rechtlichen Aspekten, insbesondere mit Blick auf die Grundrechte unverhältnismäßig und bedenklich.

\section{Maßnahmen im Bereich des Strafvollzugs}

Das bereits erwähnte 1. COVID-19-JuBG hat der BMJ auch im Bereich des Strafvollzugs verschiedene Verordnungsermächtigungen eingeräumt. Durch den Verweis auf die sinngemäße Anwendung des I. Hauptstücks des genannten Gesetzes stehen diese unter dem Leitgedanken, „einerseits die Gefahr eines Übergreifens des Virus auf den Strafvollzug möglichst hintanzuhalten, andererseits aber auch das System des Strafvollzugs, einschließlich seiner rechtsstaatlichen Garantien, möglichst aufrechtzuerhalten bzw. zu bewahren, um so bestmöglich zur Sicherheit der Allgemeinheit, aber nicht zuletzt auch der Strafvollzugsbediensteten und der im Strafvollzug befindlichen Personen beizutragen" ${ }^{37}$ Die folgenden Ausführungen betreffen beispielshaft die Möglichkeiten zum Haftaufschub sowie die Einschränkungen des Besuchsverkehrs gegenüber Gefangenen, um die Frage, inwieweit die COVID-19-Maßnahmen im Bereich des Strafrechts verhältnismäßig waren, abzurunden. ${ }^{38}$

\subsection{Möglichkeiten zum Haftaufschub}

$\$ 10 \mathrm{Z} 1$ 1. COVID-19-JuBG erlaubt es der BMJ, per Verordnung das Unterbleiben der Aufforderung zum Strafantritt für einen auf freiem Fuß befindlichen rechtskräftig zu einer Freiheitsstrafe Verurteilten $(\mathbb{3} 3$ Abs. 2 erster

37 Initiativantrag (IA) Nr. 397/A 27. GP S. 39.

$38 \mathrm{Zu}$ den anderen Änderungen im Bereich des Strafvollzugs siehe Birklbauer in Resch, Corona-HB Kap 16 Rz 79 ff. 
Satz $\mathrm{StVG}^{39}$ ) für die Dauer von COVID-19-Maßnahmen, also längstens bis 31.12.2020, anzuordnen, während $₫ 10 \mathrm{Z} 4$ 1. COVID-19-JuBG generell einen Vollzugsaufschub ( $\mathbb{6} 6$ Abs. 1 StVG) für Freiheitsstrafen bis zu drei Jahren ermöglicht, um den Zugang in den Strafvollzug zu beschränken. Darüber hinaus ermächtigt $₫ 10 \mathrm{Z} 31$. COVID-19-JuBG dazu, mit COVID-19 infizierte Personen oder solche, die wegen Kontakts mit infizierten Personen unter Quarantäne stehen, gemäß $\$ 5$ StVG als (ursprünglich) und $\$ 133$ StVG als (nachträglich) vollzugsuntauglich einzustufen. Dies bedeutet nicht, dass diese Personen in jedem Fall auf freiem Fuß bleiben oder enthaftet werden, sondern allenfalls eine Haft anderer Art (z. B. in einer öffentlichen Krankenanstalt) vollzogen werden kann, wenn die Kapazitäten der Justizanstalten zur medizinischen Behandlung und zu besonderen Quarantänemaßnahmen erschöpft sind. ${ }^{40}$ Eine solche Haft anderer Art in einer Krankenanstalt ist für den Verurteilten günstig, weil er durch seine Krankheit ohnehin in seiner Freiheit beschränkt ist und mit dem „besonderen Strafvollzug“ im Ergebnis einen Teil seiner Freiheitsstrafe auf diese Weise verbüßt.

Die Umsetzung dieser positiv zu bewertenden gesetzlichen Ermächtigungen durch die BMJ war bemerkenswert. Nach $\$ 2$ der entsprechenden Verordnung ${ }^{41}$ war zwar bei einem Verurteilten, der sich auf freiem Fuß befand, ein Strafantritt aufzuschieben ${ }^{42}$, davon wurden aber Verurteilte wegen einer in $\$ 33$ Abs. 2 StGB umschriebenen Tat (im Wesentlichen Gewalt im sozialen Nahbereich, in hohem Ausmaß oder unter Waffeneinsatz) oder sonst wegen eines bestimmten Sexualdelikts ( $\$ \$ \$ 201,202,205$, 205a, 206, 207, 207a oder 207b StGB) ausgenommen. Durch diesen Ausschluss bestimmter Tätergruppen vom Vollzugsaufschub war die Verordnung deutlich enger als es das Gesetz erforderte. Damit wurde letztlich ausgedrückt, dass solche Straftäter weniger vor COVID-19-Infektionen zu schützen sind, was unter Verhältnismäßigkeitsgesichtspunkten und mit Blick auf die Menschenwürde mehr als bedenklich ist.

39 Bundesgesetz vom 26. März 1969 über den Vollzug der Freiheitsstrafen und der mit Freiheitsentziehung verbundenen vorbeugenden Maßnahmen (Strafvollzugsgesetz - StVG); BGBl. 144/1969.

40 IA 397/A 27. GP S. 39.

41 Verordnung der BMJ über besondere Vorkehrungen im Anwendungsbereich des StVG zur Verhinderung der Verbreitung von COVID-19 vom 26.3.2020; BGBl. II 120/2020. Die Verordnung tritt nach ihrem $\$ 10$ mit 31.12.2020 außer Kraft.

42 Das ursprüngliche Aufschubdatum bis 30.4.2020 wurde mittlerweile auf 30.6.2020, in bestimmten Fällen sogar auf 31.10.2020 geändert (BGBl. II 184/2020); dazu näher Birklbauer in Resch, Corona-HB Kap 16 Rz 83/1. 


\subsection{Besuchsbeschränkungen}

Menschen in Haft sind in ihren Kontakten zur Außenwelt von vornherein stark beschränkt. Um den vorhandenen persönlichen und familiären Beziehungen überhaupt eine Chance zu geben, sind sie - auch mit Blick auf die Resozialisierung - dennoch wesentlich. Da durch Besuche ein Infektionsrisiko besteht, war die Einschränkung der Besuchsmöglichkeiten im Rahmen der COVID-19-Krise naheliegend. Vor diesem Hintergrund ermächtigte $₫ 10 \mathrm{Z} 5$ 1. COVID-19-JuBG die BMJ, den Besuchsverkehr ( $\$ 93$ StVG) für die Dauer der vorläufigen COVID-19-Maßnahmen auf telefonische Kontakte zu beschränken oder sonstige Beschränkungen des Verkehrs mit $\operatorname{der}$ Außenwelt vorzusehen, wobei sich für diese Beschränkungen keine weitere Determinierung fand. ${ }^{43}$ Mit $\ 5$ der entsprechenden Verordnung ${ }^{44}$ wurde der Besuchsverkehr ( $\$ 93 \mathrm{StVG}$ ), mit Ausnahme der Besuche von Vertretern öffentlicher Stellen und von Betreuungseinrichtungen sowie von Rechtsbeiständen ( $\$ 96 \mathrm{StVG}$ ) letztlich bis zum 10.5.202045 auf telefonische Kontakte beschränkt. Das Besuchsverbot zwischen 1. und 10.5.2020 wurde insofern gelockert, als ab diesem Zeitpunkt Besuche einzelner Personen zugelassen wurden, bei unter 14-jährigen Besuchern auch mit einer erwachsenen Begleitperson. Damit sollte einerseits gewährleistet werden, dass Infektionen von Strafgefangenen und Vollzugspersonal mit COVID-19 nach Möglichkeit verhindert werden, andererseits doch auch den Rechten von Gefangenen entsprochen werden. Insgesamt kann diese Regelung als verhältnismäßig eingestuft werden.

43 Eine inhaltsgleiche Ermächtigung sieht $₫ 9$ Z 5 1. COVID-19-JuBG für den Bereich der U-Haft ( $\$ 188$ Abs 1 StPO) vor.

44 BGBl. II 120/2020.

45 BGBl. II 184/2020. Zur inhaltsgleichen Regelung für die U-Haft siehe $₫ 5$ der Verordnung der BMJ, mit der zur Verhinderung der Verbreitung von COVID-19 besondere Vorkehrungen in Strafsachen getroffen werden (BGBl. II 113/2020 in der Fassung BGBl. II 180/2020). Die Verordnung spricht hier allerdings von „Strafgefangenen", wodurch die Anwendbarkeit der Beschränkungen auf U-Häftlinge infolge der Unschuldsvermutung (Art. 6 Abs. 2 EMRK) fraglich ist; siehe dazu Birklbauer in Resch, Corona-HB Kap 16 Rz 42/1. 


\section{Zusammenfassende Schlussfolgerungen}

Dass der Gesetzgeber keine neuen Strafbestimmungen im Bereich des gerichtlichen materiellen Strafrechts geschaffen hat, war eine Folge des Ultima-RatioGedankens und hat insofern dem Verhältnismäßigkeitsgrundsatz entsprochen. Durch die Schaffung von Strafnormen im Bereich des Verwaltungsstrafrechts hat der Gesetzgeber ausreichend reagiert, zumal durch diese Normen indirekt auch eine Strafbarkeit wegen Körperverletzungs- und Gesundheitsgefährdungsdelikten des StGB entstehen kann, wenn sich durch (vorsätzliches oder fahrlässiges) normwidriges Verhalten von infizierten oder auch nicht-infizierten Personen eine konkrete Ansteckung nachweisen lässt. Dass Verwaltungsstrafen aber tendenziell weniger sozial gerecht sind als gerichtliche Geldstrafen, soll in diesem Zusammenhang nicht unerwähnt bleiben.

Beim Strafverfahrensrecht war die Grundidee, Vernehmungen per Video zu führen, um direkte Kontakte zu vermeiden, verhältnismäßig, wenn die Verteidigungsrechte ausreichend gewahrt werden. Die ausreichende Wahrung von Verteidigungsrechten blieb aber im Rahmen der Normschaffung weitgehend unberücksichtigt. Für die zugelassene Möglichkeit, auf Verhandlungen zur Haftverlängerung zu verzichten und den Freiheitsentzug ohne Vernehmung des Inhaftierten fortzusetzen, fehlte jede Notwendigkeit. Diese Maßnahme im Bereich des Strafverfahrens ist als unverhältnismäßig und grundrechtlich bedenklich zu qualifizieren. Gleiches gilt für die faktische Beschränkung der Öffentlichkeit einer Hauptverhandlung während des Shut Downs.

Für den Strafvollzug ist das Ansinnen, Gefangene von COVID-19Infektionen zu schützen, infolge des besonderen Schutzverhältnisses des Staates gegenüber diesen Menschen grundsätzlich verhältnismäßig. Insofern waren auch die Besuchsbeschränkungen ein richtiger Weg. Strafantritte bei vertretbarem Risiko für die Gesellschaft aufzuschieben war ebenso ein richtiger Schritt. Dass aber bestimmte Straftätergruppen, die sich auf freiem Fuß befanden, was ein entsprechendes fehlendes Gefährdungspotential inkludiert, um jeden Preis sofort ins Gefängnis mussten, ist absolut unverhältnismäßig und zeugt von einem Menschenbild, das eines liberaldemokratischen Rechtsstaats, der den Grundrechten verpflichtet ist, absolut unwürdig ist.

Die Gesamtbewertung der Verhältnismäßigkeit der COVID-19Maßnahmen aus strafrechtlicher Sicht fällt somit gemischt aus. Der Weg, keine Neuerungen im materiellen Strafrecht vorzunehmen und die neuen technischen Möglichkeiten im Rahmen von Strafverfahren zu nutzen, um auch hier ein „Social Distancing“ zu ermöglichen, war jedenfalls vertret- 
bar. Freiheitsentziehungen rein nach Lage der Akten zu verlängern oder die Öffentlichkeit von Hauptverhandlungen faktisch auszuschließen, war dagegen grundrechtlich bedenklich. Das Ansinnen, bestimmte Straftätergruppen (Gewalt- und Sexualtäter) systematisch der möglichen Gefahr einer Infektion mit dem Corona-Virus auszusetzen, ist nicht nur nicht zu rechtfertigen, sondern der dahinterstehende Gedanke ist menschenverachtend und einer liberalen Demokratie unwürdig.

\section{Literatur}

Birklbauer, Alois: Die Öffentlichkeit der Hauptverhandlung. Ein Prozessgrundsatz im Spannungsfeld zwischen Beschuldigten-, Opfer- und Bevölkerungsinteresse, in: Journal für Strafrecht (JSt) 7/4 (2009) 109-117.

Birklbauer, Alois: Kapitel 16. Strafrecht, in: Resch, Reinhard (Hg): Das CoronaHandbuch. Österreichs Rechtspraxis zur aktuellen Lage, Wien: Manz 2020, 403432 (= Corona-HB Kap $16 \mathrm{Rz}$ xx).

Birklbauer, Alois: Die Auswirkung der COVID-19-Gesetze auf das Strafrecht, in: Journal für Strafrecht (JSt) 7/3 (2020) 197-203.

Burgstaller, Manfred/Fabrizy, Ernst Eugen: $\mathbb{8} 83$ StGB, in: Höpfel, Frank/Ratz, Eckart (Hg): Wiener Kommentar zum Strafgesetzbuch, Wien: Manz ${ }^{2} 2018$ (= WK ${ }^{2}$ StGB).

Burgstaller, Manfred/Schütz, Hannes: $\$ 6$ StGB, in: Höpfel, Frank/Ratz, Eckart (Hg): Wiener Kommentar zum Strafgesetzbuch, Wien: Manz ${ }^{2} 2017$ (= WK ${ }^{2}$ StGB).

Cohen, Lisa: Isolation, Quarantäne, Coronapartys. Die Anwendbarkeit der $\$ \$ 178 \mathrm{f}$ StGB bei Missachtung der COVID-19 Verkehrsbeschränkungen, in: Journal für Strafrecht (JSt) 7/3 (2020) 204-209.

Danek, Michael/Mann, Irene: $\$ 228$ StPO, in: Fuchs, Helmut/Ratz, Eckart $(\mathrm{Hg})$ : Wiener Kommentar zur Strafprozessordnung, Wien: Manz 2017 (= WK-StPO).

Leukauf, Otto/Steininger, Herbert/Tipold, Alexander: $\$ 178$ Strafgesetzbuch. Kommentar, Wien: Linde ${ }^{4} 2017$.

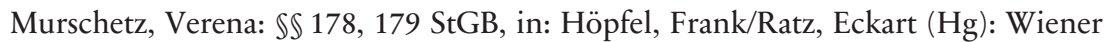
Kommentar zum Strafgesetzbuch, Wien: Manz 22020 (= WK ${ }^{2}$ StGB).

Reindl-Krauskopf, Susanne: $\$ 5$ StGB, in: Höpfel, Frank/Ratz, Eckart (Hg): Wiener Kommentar zum Strafgesetzbuch, Wien: Manz 2015 (= WK ${ }^{2}$ StGB). 